\title{
Detection of protonated formaldehyde in the prestellar core L1689B ${ }^{\star}, \star \star$
}

\author{
A. Bacmann ${ }^{1,2}$, E. García-García ${ }^{1,2}$, and A. Faure ${ }^{1,2}$ \\ 1 Univ. Grenoble Alpes, IPAG, 38000 Grenoble, France \\ e-mail: aurore.bacmann@univ-grenoble-alpes.fr \\ 2 CNRS, IPAG, 38000 Grenoble, France \\ Received 9 February 2016 / Accepted 29 February 2016
}

\section{ABSTRACT}

\begin{abstract}
Complex organic molecules (COMs) are detected in many regions of the interstellar medium, including prestellar cores. However, their formation mechanisms in cold $(\sim 10 \mathrm{~K})$ cores remain to this date poorly understood. The formyl radical HCO is an important candidate precursor for several O-bearing terrestrial COMs in cores, as an abundant building block of many of these molecules. Several chemical routes have been proposed to account for its formation: on grain surfaces, as an incompletely hydrogenated product of $\mathrm{H}$ addition to frozen-out $\mathrm{CO}$ molecules; and in the gas phase, either as the product of the reaction between $\mathrm{H}_{2} \mathrm{CO}$ and a radical or as a product of dissociative recombination of protonated formaldehyde $\mathrm{H}_{2} \mathrm{COH}^{+}$. The detection and abundance determination of $\mathrm{H}_{2} \mathrm{COH}^{+}$, if present, could provide clues as to whether this latter scenario might apply. We searched for protonated formaldehyde $\mathrm{H}_{2} \mathrm{COH}^{+}$in the prestellar core L1689B using the IRAM $30 \mathrm{~m}$ telescope. The $\mathrm{H}_{2} \mathrm{COH}^{+}$ion is unambiguously detected, for the first time, in a cold $(\sim 10 \mathrm{~K})$ source. The derived abundance agrees with a scenario in which the formation of $\mathrm{H}_{2} \mathrm{COH}^{+}$results from the protonation of formaldehyde. We use this abundance value to constrain the branching ratio of the dissociative recombination of $\mathrm{H}_{2} \mathrm{COH}^{+}$towards the $\mathrm{HCO}$ channel to $\sim 10-30 \%$. This value could however be lower if $\mathrm{HCO}$ were efficiently formed from neutralneutral reactions in the gas phase, and we stress the need for laboratory measurements of the rate constants of these reactions at $10 \mathrm{~K}$. Given the experimental difficulties in measuring branching ratios experimentally, observations can place valuable constraints on these values and provide useful input for chemical networks.
\end{abstract}

Key words. ISM: molecules - line: identification - ISM: abundances

\section{Introduction}

Despite their low temperatures, prestellar cores harbour a wealth of chemical species. In recent years, complex organic molecules (COMs), which were previously thought to trace mainly warm gas in star-forming regions, have been detected in prestellar sources (e.g. Bacmann et al. 2012; Vastel et al. 2014) where the temperatures are around $10 \mathrm{~K}$. The formation mechanisms of the terrestrial COMs, (i.e. which are stable under Earth-like conditions) currently detected in the cold gas remain poorly understood, and the respective roles of gas-phase reactions or grain surface chemistry are still being debated.

Radicals like the formyl radical HCO or the methoxy radical $\mathrm{CH}_{3} \mathrm{O}$ have drawn attention as the possible precursors of COMs, either in the gas phase (Vasyunin \& Herbst 2013; Balucani et al. 2015) or on grain surfaces (Garrod \& Herbst 2006). They are also important intermediates in the grain-surface synthesis of methanol, as products of $\mathrm{H}$-atom additions to $\mathrm{CO}$ (e.g. Brown et al. 1988; Pirim \& Krim 2011). These radicals are also widely detected in the gas phase of prestellar cores and cold clouds (Bacmann \& Faure 2016; Agúndez et al. 2015b; Gerin et al. 2009; Cernicharo et al. 2012).

\footnotetext{
* Based on observations carried out with the IRAM $30 \mathrm{~m}$ telescope. IRAM is supported by INSU/CNRS (France), MPG (Germany), and IGN (Spain).

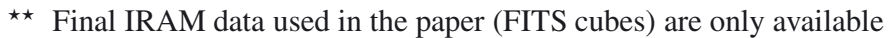
at the CDS via anonymous ftp to cdsarc.u-strasbg. fr $(130.79 .128 .5)$ or via

http://cdsarc.u-strasbg.fr/viz-bin/qcat?]/A+A/588/L8
}

In a previous survey of these radicals in a sample of prestellar cores, Bacmann \& Faure (2016) proposed that the HCO abundances measured in the gas phase could be accounted for by a pure gas-phase scenario, in which HCO results from the dissociative recombination of protonated formaldehyde $\mathrm{H}_{2} \mathrm{COH}^{+}$. In dark clouds, $\mathrm{H}_{2} \mathrm{COH}^{+}$is likely the product of the protonation of $\mathrm{H}_{2} \mathrm{CO}$, which is an abundant organic species in prestellar cores, with abundances generally around $10^{-10}-10^{-9}$ (Bacmann et al. 2003; Guzmán et al. 2011). Proton donors, such as $\mathrm{H}_{3}^{+}$, or $\mathrm{HCO}^{+}$, are the most abundant ions, with abundances close to $10^{-9}-10^{-8}$ (Aikawa et al. 2005; Flower et al. 2005, 2006). It is therefore expected that protonated formaldehyde $\mathrm{H}_{2} \mathrm{COH}^{+}$ would easily be formed. Previous searches by Minh et al. (1993) towards Orion A and the two cold clouds L183 and TMC-1 yielded no detection, and Ohishi et al. (1996) detected $\mathrm{H}_{2} \mathrm{COH}^{+}$ only towards Sgr B2 and several hot cores and not towards the cold sources of their sample.

In this Letter, we present the first detection of protonated formaldehyde in a cold core, and discuss its abundance in terms of the formation of the HCO radical by ion-molecule chemistry, and the possibility to constrain the branching ratio towards $\mathrm{HCO}$ of its dissociative recombination with electrons.

\section{Observations and data analysis}

The frequencies of the rotational transitions of $\mathrm{H}_{2} \mathrm{COH}^{+}$were determined by Chomiak et al. (1994) and Dore et al. (1995) and were retrieved from the CDMS spectroscopy catalogue (Müller et al. 2001, 2005). Line excitation is always an issue in cold $(\sim 10 \mathrm{~K})$ gas and only levels with a low energy can be populated. To search for $\mathrm{H}_{2} \mathrm{COH}^{+}$, we therefore selected transitions with 
Table 1. Observed $\mathrm{H}_{2} \mathrm{COH}^{+}$transitions.

\begin{tabular}{lcccl}
\hline \hline $\begin{array}{l}\text { Transition } \\
J_{K_{\mathrm{a}} K_{\mathrm{c}}}\end{array}$ & $\begin{array}{c}\text { Frequency } \\
\mathrm{MHz}\end{array}$ & $\begin{array}{c}E_{\mathrm{up}} \\
\mathrm{K}\end{array}$ & $\begin{array}{l}g_{\text {up }} \\
\mathrm{A}_{\mathrm{ul}} \\
\mathrm{s}^{-1}\end{array}$ \\
\hline $4_{04}-3_{13}$ & 102065.86 & 30.4 & 9 & $7.27 \times 10^{-6}$ \\
$2_{02}-1_{11}$ & 126923.38 & 9.1 & 5 & $1.83 \times 10^{-5}$ \\
$2_{11}-1_{10}$ & 132219.70 & 17.5 & 5 & $1.55 \times 10^{-5}$ \\
$1_{10}-1_{01}$ & 168401.14 & 11.1 & 3 & $8.77 \times 10^{-5}$ \\
\hline
\end{tabular}

upper level energies lower than $\sim 20 \mathrm{~K}$, which could be observed in a minimum of frequency setups, and for which good atmospheric transmission did not require very dry weather conditions. The chosen three lines at $2 \mathrm{~mm}$ and their spectroscopic parameters are shown in Table 1, to which we added a line at $3 \mathrm{~mm}$ from a previous project.

The observations were carried out towards the prestellar core L1689B in January and March 2012 for the $3 \mathrm{~mm}$ transition and in March 2015 for the $2 \mathrm{~mm}$ transitions with the IRAM $30 \mathrm{~m}$ telescope located at Pico Veleta, Spain. The source was selected on the grounds that its molecular lines are usually stronger than in other similar sources (Bacmann \& Faure 2016). The integration coordinates were $\alpha_{2000}=16^{\mathrm{h}} 34^{\mathrm{m}} 48.30^{\mathrm{s}}$ and $\delta_{2000}=-24^{\circ} 38^{\prime} 04.0^{\prime \prime}$, corresponding to the peak of the millimetre dust continuum emission. We used the receivers E090 and E150 operating at $3 \mathrm{~mm}$ and $2 \mathrm{~mm}$, respectively, which were connected to the Fourier transform spectrometer (FTS) at a frequency resolution of $\sim 50 \mathrm{kHz}$; this corresponded to velocity resolutions of $0.15 \mathrm{~km} \mathrm{~s}^{-1}$ at $102 \mathrm{GHz}, 0.11 \mathrm{~km} \mathrm{~s}^{-1}$ at $130 \mathrm{GHz}$, and $0.09 \mathrm{~km} \mathrm{~s}^{-1}$ at $170 \mathrm{GHz}$. The weather conditions were good during the 2012 observing runs, and excellent during the March 2015 run with precipitable water vapour of $1 \mathrm{~mm}$ on average and system temperatures of $\sim 80 \mathrm{~K}$ at $126-132 \mathrm{GHz}$ and $\sim 130 \mathrm{~K}$ at $170 \mathrm{GHz}$. Pointing was checked every $1.5 \mathrm{~h}$ on a nearby quasar and found to be within $2-3^{\prime \prime}$ at $2 \mathrm{~mm}$ and $3-4^{\prime \prime}$ at $3 \mathrm{~mm}$. Focus was performed on a strong quasar at the beginning of each observing session and on Mercury after sunrise. The data were taken with the frequency switching mode with a frequency throw of 7.5 MHz. The antenna forward efficiency $F_{\text {eff }}$ is 0.95 and 0.93 at $3 \mathrm{~mm}$ and $2 \mathrm{~mm}$, respectively, and the main beam efficiencies $B_{\text {eff }}$ were taken to be $0.79,0.77,0.76$, and 0.70 at $106.1 \mathrm{GHz}$, $126.9 \mathrm{GHz}, 132.2 \mathrm{GHz}$, and $168.4 \mathrm{GHz}$, respectively. From integrations carried out at different offsets (which is discussed elsewhere), we find that the emission fills the main beam but is not very extended, and therefore we used the main beam temperature scale in our analysis, applying $T_{\mathrm{mb}}=F_{\text {eff }} / B_{\text {eff }} T_{\mathrm{a}}^{*}$. The main beam size is $24^{\prime \prime}$ at $106.1 \mathrm{GHz}, 19^{\prime \prime}$ at $126.9 \mathrm{GHz}$ and at $132.2 \mathrm{GHz}$, and $15^{\prime \prime}$ at $168.4 \mathrm{GHz}$.

The data were reduced using the IRAM GILDAS/CLASS ${ }^{1}$ software: the individual scans were averaged together and a loworder (typically 3 ) polynomial was fitted to line-free regions of the spectra to subtract a baseline. The resulting spectra were then folded to recover from the effect of the frequency switching procedure.

\section{Column density determination}

Protonated formaldehyde is clearly detected, as can be seen from the spectra shown in Fig. 1. The transition at $102 \mathrm{GHz}$ is however not detected, which can be explained by its lower Einstein spontaneous emission coefficient and higher upper level energy. The line parameters, velocity integrated intensities, line widths, and peak line intensities, were determined by fitting a Gaussian to each line. The obtained values are presented in Table 2.

\footnotetext{
1 http://www .iram. fr/IRAMFR/GILDAS
}

Table 2. Line parameters for the observed $\mathrm{H}_{2} \mathrm{COH}^{+}$transitions.

\begin{tabular}{lcccc}
\hline \hline $\begin{array}{l}\text { Frequency } \\
\mathrm{MHz}\end{array}$ & $\begin{array}{c}\mathrm{rms} \\
\mathrm{mK}\end{array}$ & $\begin{array}{c}T_{\mathrm{mb}} \\
\mathrm{mK}\end{array}$ & $\begin{array}{c}\Delta v \\
\mathrm{~km} \mathrm{~s}^{-1}\end{array}$ & $\begin{array}{c}\text { Integrated intensity } \\
\mathrm{K} \mathrm{km} \mathrm{s}^{-1}\end{array}$ \\
\hline 102065.86 & 3.3 & - & - & $<0.0024$ \\
126923.38 & 6.0 & 116 & $0.45(0.02)$ & $0.0553(0.0085)$ \\
132219.70 & 2.6 & 12 & $0.35(0.06)$ & $0.0043(0.0010)$ \\
168401.14 & 7.6 & 67 & $0.40(0.03)$ & $0.0290(0.0048)$ \\
\hline
\end{tabular}

Notes. The rms is given for $50 \mathrm{kHz}$ channels. The numbers between parenthesis are the $1 \sigma$ uncertainties. The upper limit is $3 \sigma$.

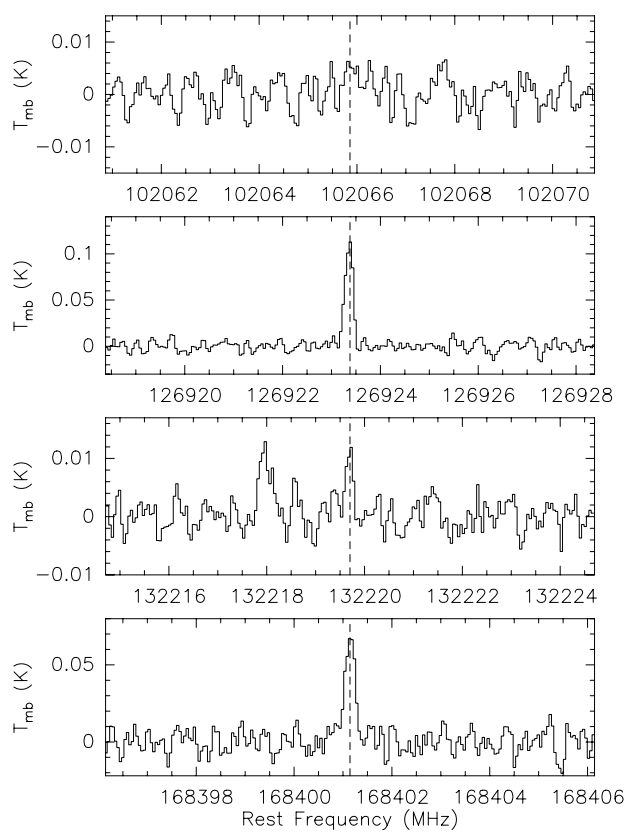

Fig. 1. $\mathrm{H}_{2} \mathrm{COH}^{+}$spectra in L1689B. The vertical dashed lines indicate the positions of the $\mathrm{H}_{2} \mathrm{COH}^{+}$transitions. The feature at $\sim 132218 \mathrm{MHz}$ does not correspond to any line from the CDMS or the JPL catalogue.

There are no available collisional coefficients for $\mathrm{H}_{2} \mathrm{COH}^{+}$, so we perform a simple derivation of the column density assuming local thermodynamic equilibrium (LTE). The method we use has been described in Bacmann \& Faure (2016). Briefly, we calculate the integrated intensities of the transitions under the LTE assumption for a range of column density and excitation temperature values, and perform a least-squares fit, defining a $\chi^{2}$ between the calculated integrated intensities and observed integrated intensities for the detected lines.

The best model yields a column density of $6.7 \times 10^{11} \mathrm{~cm}^{-2}$ and a temperature of $4.2 \mathrm{~K}$. Reasonably good fits (i.e. for which $\chi^{2}$ is within $1 \sigma$ of the minimum $\chi^{2}$ value) can be found for high values of the column density $\left(\gtrsim 1.5 \times 10^{12} \mathrm{~cm}^{-2}\right)$, but these fits are obtained for excitation temperatures that are below $3.5 \mathrm{~K}$. We limit ourselves to excitation temperatures above $3.7 \mathrm{~K}$ because lower values become unrealistic. With this additional condition, we find that the beam averaged $\mathrm{H}_{2} \mathrm{COH}^{+}$column density is between $3.3 \times 10^{11} \mathrm{~cm}^{-2}$ and $1.1 \times 10^{12} \mathrm{~cm}^{-2}$. The non-detection of the $102 \mathrm{GHz}$ line does not bring supplementary constraints despite the high sensitivity of the observation because the low excitation temperatures considered $(<5 \mathrm{~K})$ are compatible with a non-detection of this line even for unrealistically high column densities, for which the other lines would be stronger and optically thick. Despite the low number of lines in our analysis, the best fit is only moderately good, as shown in Fig. 2. This probably means that the excitation deviates from LTE. 


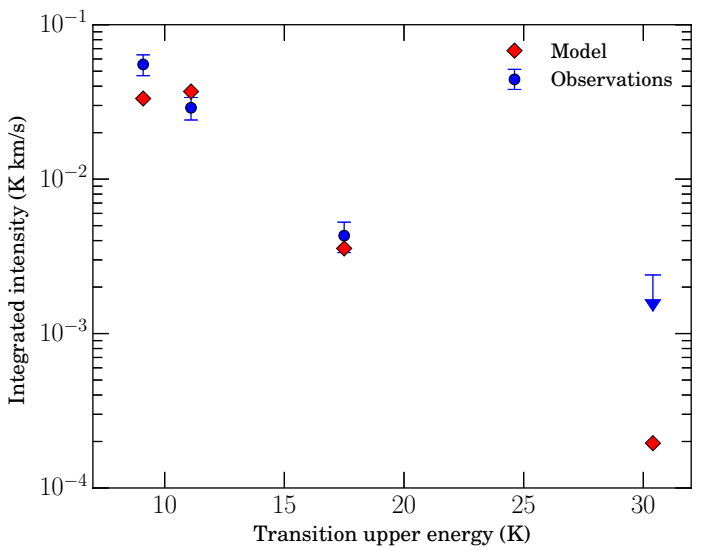

Fig. 2. Comparison of modelled and observed integrated intensities for the four considered $\mathrm{H}_{2} \mathrm{COH}^{+}$transitions.

\section{Discussion and conclusions}

This detection represents the first detection of protonated formaldehyde in a cold prestellar core. Former searches for this ion in similar sources did not yield any detection, most probably because of lack of sensitivity in the observations. The spectral resolution of the $\mathrm{H}_{2} \mathrm{COH}^{+}$non-detections in the cold sources of Ohishi et al. (1996) is ambiguous. If we assume that it was $250 \mathrm{kHz}$, the rms noise of $20 \mathrm{mK}$ reached by Ohishi et al. (1996) was not low enough to detect the $168.4 \mathrm{GHz}$ line if it was as strong as in L1689B.

Most likely, the dominant reaction route to form $\mathrm{H}_{2} \mathrm{COH}^{+}$in dark clouds is the protonation of formaldehyde

$\mathrm{H}_{2} \mathrm{CO}+\mathrm{HX}^{+} \stackrel{k_{\mathrm{f}}}{\rightarrow} \mathrm{H}_{2} \mathrm{COH}^{+}+\mathrm{H}_{2}$,

where $\mathrm{HX}^{+}$stands for a proton donor. Indeed, both $\mathrm{H}_{2} \mathrm{CO}$ and proton donors (e.g. $\mathrm{H}_{3}^{+}$, its deuterated isotopologues, or $\mathrm{HCO}^{+}$) are abundant in prestellar cores, and exothermic ion-molecule processes are generally fast. Bacmann \& Faure (2016) estimated the rate for reaction (1) with $\mathrm{HX}^{+}=\mathrm{H}_{3}^{+}$to be $k_{\mathrm{f}}=$ $7 \times 10^{-8} \mathrm{~cm}^{3} \mathrm{~s}^{-1}$ at $10 \mathrm{~K}$, using the locked dipole approximation. Because of the difference in reduced mass, this rate constant becomes $k_{\mathrm{f}}=3.1 \times 10^{-8} \mathrm{~cm}^{3} \mathrm{~s}^{-1}$ at $10 \mathrm{~K}$ if $\mathrm{HX}^{+}=\mathrm{HCO}^{+}$. The most efficient destruction route for $\mathrm{H}_{2} \mathrm{COH}^{+}$is the dissociative recombination (DR) with electrons

$\mathrm{H}_{2} \mathrm{COH}^{+}+\mathrm{e}^{-} \stackrel{k_{\mathrm{d}}}{\rightarrow}$ products.

Reaction (2) was studied experimentally by Hamberg et al. (2007, and recently by Osborne et al. 2015) who determined $k_{\mathrm{d}}=9.9 \times 10^{-6} \mathrm{~cm}^{3} \mathrm{~s}^{-1}$ at $10 \mathrm{~K}$. At steady state, if $\mathrm{H}_{2} \mathrm{COH}^{+}$ is indeed formed mostly by reaction (1) and destroyed by reaction (2), its abundance is simply given by

$\left[\mathrm{H}_{2} \mathrm{COH}^{+}\right]=\frac{k_{\mathrm{f}}}{k_{\mathrm{d}}} \frac{\left[\mathrm{HX}^{+}\right]}{\left[\mathrm{e}^{-}\right]}\left[\mathrm{H}_{2} \mathrm{CO}\right]$

Substituting the reaction rates with their values for $\mathrm{H}_{3}^{+}$and assuming the proton donor abundance is equal to the electron abundance, we predict an abundance of $\left[\mathrm{H}_{2} \mathrm{COH}^{+}\right] \approx$ $0.007\left[\mathrm{H}_{2} \mathrm{CO}\right]$ as mentioned in Bacmann \& Faure (2016), or $\left[\mathrm{H}_{2} \mathrm{COH}^{+}\right] \approx 0.003\left[\mathrm{H}_{2} \mathrm{CO}\right]$ if $\mathrm{HCO}^{+}$is the main proton donor. Using an $\mathrm{H}_{2} \mathrm{CO}$ column density of $1.3 \times 10^{14} \mathrm{~cm}^{-2}$ (Bacmann et al. 2003), we find that the predicted $\mathrm{H}_{2} \mathrm{COH}^{+}$column density $N^{\text {mod }}$ derived from Eq. (3) is $4.1 \times 10^{11}-9.1 \times 10^{11} \mathrm{~cm}^{-2}$ (depending on the main proton donor), which is consistent with the observed value we determine in L1689B, $N^{\text {obs }}=6.7 \times 10^{11} \mathrm{~cm}^{-2}$ within the uncertainties. This also nicely confirms the value of the destruction rate of $\mathrm{H}_{2} \mathrm{COH}^{+}$measured by Hamberg et al. (2007) at $10 \mathrm{~K}$.

Agúndez et al. (2015a) have run a time-dependent chemical model using the UMIST12 network (McElroy et al. 2013) and derive $\left[\mathrm{H}_{2} \mathrm{COH}^{+}\right] \sim 8 \times 10^{-4}-10^{-3}\left[\mathrm{H}_{2} \mathrm{CO}\right]$, about six times smaller than our observed value. Though our model better reproduces the observations, it considers only one formation and one destruction route for $\mathrm{H}_{2} \mathrm{COH}^{+}$. The $\mathrm{H}_{2} \mathrm{CO}$ protonation reactions in the UMIST12 network producing $\mathrm{H}_{2} \mathrm{COH}^{+}$have smaller reaction rates than the rate we use (a factor of 2 ). Our model might also overestimate the amount of proton donors reacting with $\mathrm{H}_{2} \mathrm{CO}$, since we assume it equal to the electron abundance. We also neglect other destruction routes than electronic DR for $\mathrm{H}_{2} \mathrm{COH}^{+}$, such as proton transfer between $\mathrm{H}_{2} \mathrm{COH}^{+}$and $\mathrm{CH}_{3} \mathrm{OH}$ or $\mathrm{NH}_{3}$, but these are about 1000 times slower than DR at $10 \mathrm{~K}$ and unlikely to be a cause for the discrepancy between both models.

The dissociative recombination of $\mathrm{H}_{2} \mathrm{COH}^{+}$with electrons has several output channels. According to the experiments by Hamberg et al. (2007), the products of the reaction are

$$
\begin{aligned}
\mathrm{H}_{2} \mathrm{COH}^{+}+\mathrm{e}^{-} & \rightarrow \mathrm{HCO}+x \mathrm{H}+y \mathrm{H}_{2} \\
& \rightarrow \mathrm{CO}+x \mathrm{H}+y \mathrm{H}_{2} \\
& \rightarrow \mathrm{H}_{2} \mathrm{CO}+\mathrm{H} \\
& \rightarrow \mathrm{CH}_{2}+\mathrm{OH} \\
& \rightarrow \mathrm{CH}+\mathrm{H}_{2} \mathrm{O}
\end{aligned}
$$

where $x$ and $y$ are integers that account for the different possible combinations of $\mathrm{H}$ and $\mathrm{H}_{2}$ in the products. The experimental branching ratios are $6 \%$ for $\mathrm{CH}_{2}, 2 \%$ for $\mathrm{CH}$, and $92 \%$ for the channels where the $\mathrm{C}-\mathrm{O}$ bond is conserved (i.e. $\mathrm{CO}, \mathrm{HCO}$, and $\mathrm{H}_{2} \mathrm{CO}$ ). This is in contrast to the dissociative recombination of $\mathrm{CH}_{3} \mathrm{OH}$ for which the $\mathrm{C}-\mathrm{O}$ bond is preserved in a minority of channels (Geppert et al. 2006). In the case of $\mathrm{H}_{2} \mathrm{COH}^{+}$, the experiment by Hamberg et al. (2007) did not distinguish between $\mathrm{HCO}, \mathrm{CO}$, and $\mathrm{H}_{2} \mathrm{CO}$.

In order to account for the gas-phase abundance of the $\mathrm{HCO}$ radical in a sample of prestellar cores, and in particular the constant abundance ratio of $\sim 10$ between $\mathrm{H}_{2} \mathrm{CO}$ and HCO, Bacmann \& Faure (2016) suggested that the observed $\mathrm{HCO} / \mathrm{H}_{2} \mathrm{CO}$ abundance ratio can be reproduced if $\mathrm{HCO}$ originates from the dissociative recombination of $\mathrm{H}_{2} \mathrm{COH}^{+}$, assuming that the branching ratio of the DR is $\sim 10 \%$ for the $\mathrm{HCO}$ channel (reaction (4) above). Although the detection of $\mathrm{H}_{2} \mathrm{COH}^{+}$ in a prestellar core does not provide unambiguous evidence that $\mathrm{HCO}$ forms from the protonation of formaldehyde followed by dissociative recombination, it is still in agreement with this scenario, and allows us to directly constrain the branching ratio of the dissociative recombination for the HCO channel. In this framework, and assuming the main destruction route for $\mathrm{HCO}$ is with a proton donor like $\mathrm{H}_{3}^{+}$, the branching ratio $f$ for $\mathrm{HCO}$ is given by

\section{$k_{\mathrm{dd}}[\mathrm{HCO}]\left[\mathrm{H}_{3}^{+}\right]=f k_{\mathrm{d}}\left[\mathrm{H}_{2} \mathrm{COH}^{+}\right]\left[\mathrm{e}^{-}\right]$,}

where $k_{\mathrm{dd}}=5 \times 10^{-8} \mathrm{~cm}^{3} \mathrm{~s}^{-1}$ is the destruction rate of $\mathrm{HCO}$ with $\mathrm{H}_{3}^{+}$at $10 \mathrm{~K}$ (Bacmann \& Faure 2016). Assuming as before $\left[\mathrm{H}_{3}^{+}\right] \approx\left[\mathrm{e}^{-}\right]$, the $\mathrm{H}_{2} \mathrm{COH}^{+}$column density determined in this study $\left(N_{\mathrm{H}_{2} \mathrm{COH}^{+}}^{\text {obs }}=6.7 \times 10^{11} \mathrm{~cm}^{-2}\right)$ and the $\mathrm{HCO}$ column density in L1689B given in Bacmann \& Faure (2016) $\left(N_{\mathrm{HCO}}=1.3 \times\right.$ $10^{13} \mathrm{~cm}^{-2}$ ), we find again $f \sim 10 \%$, without invoking the $\mathrm{H}_{2} \mathrm{CO}$ abundance. 
As discussed in Bacmann \& Faure (2016), another potential destruction route for $\mathrm{HCO}$ is with abundant atoms such as $\mathrm{H}$. In this case, assuming an atomic $\mathrm{H}$ abundance of $\sim 10^{-5}$ with respect to $\mathrm{H}_{2}$, an electronic abundance of $10^{-8}$, and $k_{\mathrm{H}}=$ $1.5 \times 10^{-10} \mathrm{~cm}^{3} \mathrm{~s}^{-1}$ for the rate constant of the reaction $\mathrm{HCO}+$ $\mathrm{H}$ (the value at $300 \mathrm{~K}$ from Baulch et al. 2005), we find a value for the branching ratio $f$ of $30 \%$. It is however unclear whether the value of $k_{\mathrm{H}}$ taken here also applies at $10 \mathrm{~K}$, since no measurements of this rate constant are available at low temperatures.

One major uncertainty in this determination of the branching ratio results from possible alternative formation scenarios for $\mathrm{HCO}$, which could be non-negligible. One such route is the neutral-neutral reaction between $\mathrm{H}_{2} \mathrm{CO}$ and an abundant radical such as $\mathrm{OH}$ or $\mathrm{CN}$. Recent experimental studies have confirmed that such reactions could proceed efficiently via tunnelling at low temperatures. Indeed, Shannon et al. (2013) and Gómez Martín et al. (2014) have shown that the reaction $\mathrm{CH}_{3} \mathrm{OH}+\mathrm{OH}$ gets faster at low temperatures down to $\sim 50 \mathrm{~K}$. A spectacular increase in the reaction constant is also reported by Jiménez et al. (2016) for $\mathrm{CH}_{3} \mathrm{OCHO}+\mathrm{OH}$; the reaction rate increases by three orders of magnitude between $300 \mathrm{~K}$ and $22 \mathrm{~K}$ and by an order of magnitude between $60 \mathrm{~K}$ and $22 \mathrm{~K}$, reaching as high a value as $1.2 \times 10^{-10} \mathrm{~cm}^{3} \mathrm{~s}^{-1}$. In order to be as efficient as the ion-molecule route to form $\mathrm{HCO}$, the constant of the reaction between $\mathrm{H}_{2} \mathrm{CO}$ and $\mathrm{OH}$ would have to be $4 \times 10^{-10} \mathrm{~cm}^{3} \mathrm{~s}^{-1}$ at $10 \mathrm{~K}$ (Bacmann \& Faure 2016). No measurements at $10 \mathrm{~K}$ of this reaction, or of reactions similar to this, are available, but they are needed because the behaviour of the reaction constant is not known below $230 \mathrm{~K}$ (at which temperature it is $10^{-11} \mathrm{~cm}^{3} \mathrm{~s}^{-1}$ ) and extrapolation is hazardous. In the case that $\mathrm{H}_{2} \mathrm{CO}+\mathrm{OH}$ would be a major formation channel for $\mathrm{HCO}$, the observed $\mathrm{HCO}$ abundance could be accounted for without the need for the DR of $\mathrm{H}_{2} \mathrm{COH}^{+}$to yield a significant amount of $\mathrm{HCO}$. In this respect, the branching ratio we determined above can be considered an upper limit ${ }^{2}$.

This result can have implications for chemical networks because they assume statistical weights for the three products $\mathrm{CO}$, $\mathrm{HCO}$, and $\mathrm{H}_{2} \mathrm{CO}(1 / 3,1 / 3,1 / 3)$, as in the UMIST12 network or the KIDA ${ }^{3}$ database (Wakelam et al. 2012), following Prasad \& Huntress (1980). As already noted in Hamberg et al. (2007), these branching ratios are still vastly in agreement with their experimental results, which state that $\mathrm{CO}, \mathrm{HCO}$ and $\mathrm{H}_{2} \mathrm{CO}$ represent over $90 \%$ of the products. However, the branching ratio that is needed here to account for the observed $\mathrm{HCO}$ and $\mathrm{H}_{2} \mathrm{COH}^{+}$ abundances could be significantly different from the statistical value, as it could be $10 \%$, or lower.

This value should however be taken with some caution because the excitation of $\mathrm{H}_{2} \mathrm{COH}^{+}$is not constrained well in the absence of collisional coefficients. Our estimation of the branching ratio should therefore be repeated once collisional coefficients for $\mathrm{H}_{2} \mathrm{COH}^{+}$become available. Observations of $\mathrm{H}_{2} \mathrm{COH}^{+}$ in other prestellar sources are also needed to confirm the current findings.

To conclude, we report the detection of protonated formaldehyde $\mathrm{H}_{2} \mathrm{COH}^{+}$in the prestellar source L1689B. The derived beam-averaged column density is $6.7 \times 10^{11} \mathrm{~cm}^{-2}$, corresponding to an abundance of $\sim 1.9 \times 10^{-11}$ with respect to $\mathrm{H}_{2}$, if we

\footnotetext{
2 We also note that the neutral-neutral reaction $\mathrm{CH}_{2}+\mathrm{O}$ should yield negligible amounts of $\mathrm{HCO}$ in the conditions prevailing in cold cores (see KIDA datasheet on this reaction). Using the rate constant in the KIDA database $\left(2 \times 10^{-12} \mathrm{~cm}^{3} \mathrm{~s}^{-1}\right)$ and the steady-state abundances of $\mathrm{CH}_{2}$ and $\mathrm{O}$ from the model of Le Gal et al. (2014), we find that this reaction is 100 times less efficient than the $\mathrm{DR}$ of $\mathrm{H}_{2} \mathrm{COH}^{+}$at forming HCO.

3 http://kida.obs.u-bordeaux1.fr
}

assume an $\mathrm{H}_{2}$ column density of $3.6 \times 10^{22} \mathrm{~cm}^{-2}$ (Roy et al. 2014). It is likely however that this abundance is overestimated, as the $\mathrm{H}_{2}$ column density in Roy et al. (2014) is averaged over a $36^{\prime \prime}$ beam and is probably higher in the 15-19" beam of our $\mathrm{H}_{2} \mathrm{COH}^{+}$observations. The $\mathrm{H}_{2} \mathrm{COH}^{+}$column density agrees with the destruction rate of $\mathrm{H}_{2} \mathrm{COH}^{+}$by dissociative recombination as measured by Hamberg et al. (2007), supposing that $\mathrm{H}_{2} \mathrm{COH}^{+}$is mostly formed by protonation of $\mathrm{H}_{2} \mathrm{CO}$ in prestellar cores and destroyed by electronic dissociative recombination. Using previous observations of the radical $\mathrm{HCO}$ in the same source, we constrain the branching ratio of $\mathrm{H}_{2} \mathrm{COH}^{+}$to $\mathrm{HCO}$ to be around $10-30 \%$ or lower if $\mathrm{HCO}$ is significantly formed by gas-phase reactions between $\mathrm{H}_{2} \mathrm{CO}$ and a radical. The experimental determination of branching ratios is a fundamental piece of data in astrochemistry, but in case these measurements are not available, observations can place valuable constraints on the branching ratios, as demonstrated here.

Acknowledgements. This work has benefitted from the support of the CNRS programme "Physique et Chimie du Milieu Interstellaire" (PCMI). E. GarcíaGarcía acknowledges support from an Alpes Grenoble Innovation Recherche (AGIR) grant.

\section{References}

Agúndez, M., Cernicharo, J., de Vicente, P., et al. 2015a, A\&A, 579, L10 Agúndez, M., Cernicharo, J., \& Guélin, M. 2015b, A\&A, 577, L5

Aikawa, Y., Herbst, E., Roberts, H., \& Caselli, P. 2005, ApJ, 620, 330 Bacmann, A., \& Faure, A. 2016, A\&A, 587, A130

Bacmann, A., Lefloch, B., Ceccarelli, C., et al. 2003, ApJ, 585, L55

Bacmann, A., Taquet, V., Faure, A., Kahane, C., \& Ceccarelli, C. 2012, A\&A, $541, \mathrm{~L} 12$

Balucani, N., Ceccarelli, C., \& Taquet, V. 2015, MNRAS, 449, L16

Baulch, D. L., Bowman, C. T., Cobos, C. J., et al. 2005, J. Phys. Chem. Ref. Data, 34, 757

Brown, P. D., Charnley, S. B., \& Millar, T. J. 1988, MNRAS, 231, 409

Cernicharo, J., Marcelino, N., Roueff, E., et al. 2012, ApJ, 759, L43

Chomiak, D., Taleb-Bendiab, A., Civiš, S., \& Amano, T. 1994, Can. J. Phys., 72, 1078

Dore, L., Cazzoli, G., Civiš, S., \& Scappini, F. 1995, Chem. Phys. Lett., 244, 145 Flower, D. R., Pineau Des Forêts, G., \& Walmsley, C. M. 2005, A\&A, 436, 933 Flower, D. R., Pineau Des Forêts, G., \& Walmsley, C. M. 2006, A\&A, 449, 621 Garrod, R. T., \& Herbst, E. 2006, A\&A, 457, 927

Geppert, W. D., Hamberg, M., Thomas, R. D., et al. 2006, Faraday Discuss., 133, 177

Gerin, M., Goicoechea, J. R., Pety, J., \& Hily-Blant, P. 2009, A\&A, 494, 977

Gómez Martín, J., Caravan, R., Blitz, M., Heard, D., \& Plane, J. 2014, J. Phys. Chem. A, 118, 2693

Guzmán, V., Pety, J., Goicoechea, J. R., Gerin, M., \& Roueff, E. 2011, A\&A, 534, A49

Hamberg, M., Geppert, W. D., Thomas, R. D., et al. 2007, Mol. Phys., 105, 899 Jiménez, E., Antiñolo, M., Ballesteros, B., Canosa, A., \& Albaladejo, J. 2016, Phys. Chem. Chem. Phys., 18, 2183

Le Gal, R., Hily-Blant, P., Faure, A., et al. 2014, A\&A, 562, A83

McElroy, D., Walsh, C., Markwick, A. J., et al. 2013, A\&A, 550, A36

Minh, Y. C., Irvine, W. M., \& McGonagle, D. 1993, J. Kor. Astron. Soc., 26, 99 Müller, H. S. P., Thorwirth, S., Roth, D. A., \& Winnewisser, G. 2001, A\&A, 370, L49

Müller, H. S. P., Schlöder, F., Stutzki, J., \& Winnewisser, G. 2005, J. Mol. Struct., 742,215

Ohishi, M., Ishikawa, S.-I., Amano, T., et al. 1996, ApJ, 471, L61

Osborne, Jr., D. S., Lawson, P. A., \& Adams, N. G. 2015, Int. J. Mass Spectr., 378,193

Pirim, C., \& Krim, L. 2011, Chem. Phys., 380, 67

Prasad, S. S., \& Huntress, Jr., W. T. 1980, ApJS, 43, 1

Roy, A., André, P., Palmeirim, P., et al. 2014, A\&A, 562, A138

Shannon, R. J., Blitz, M. A., Goddard, A., \& Heard, D. E. 2013, Nature Chem., 5,745

Vastel, C., Ceccarelli, C., Lefloch, B., \& Bachiller, R. 2014, ApJ, 795, L2

Vasyunin, A. I., \& Herbst, E. 2013, ApJ, 769, 34

Wakelam, V., Herbst, E., Loison, J.-C., et al. 2012, ApJS, 199, 21 